\title{
A Discrete Time Markov Chain Model for the Assessment of Inflation Rate in Pakistan
}

\author{
Umair Arif ${ }^{1} \quad$ Muhammad Naeem Shahid ${ }^{2}$ \\ 1.Lecturer, School of Management Studies, The University of Faisalabad \\ 2.Assistant Professor, School of Management Studies, The University of Faisalabad
}

\begin{abstract}
Markov chains epitomize a class of stochastic process for a wide range of applications. Specifically, discrete time Markov chains (DTMC) is employed to model the transition probabilities between discrete states with the help of the matrices. To examine and forecast the time series the Markov chain model is applied. The most important indicator in macroeconomics is inflation, which persisted in double digits in 1970s and also in last several years. Different states are checked with the model by using inflation rate data form July 2000 to April 2015. A simulation technique used for random sequences of inflation predefine states for one year and take $1^{\text {st }}$ quarter data from it and then model the estimates by maximum likelihood and maximum likelihood with Laplace smoothing methods and check the equilibrium distribution using both techniques. Estimates obtained by Laplace Smoothing technique are reliable because it control the variation on the Maximum Likelihood estimates.
\end{abstract}

Keywords: Discrete Time Markov Chains, Maximum likelihood, Laplace Smoothing methods, Equilibrium distribution, Inflation

DOI: $10.7176 / \mathrm{MTM} / 9-5-03$

Publication date:May $31^{\text {st }} 2019$

\section{Introduction}

Discrete Markov chain models are valuable for demonstrating mostly applied structures such as trade systems, line up systems [II] and account systems [III]. These models are used for displaying definite data series can also be found in mostly real world areas [IV]. Time series are used often in mostly real world areas. If anyone modeled the time series precisely, then it is easy to make accurate estimates and also optimally forecast in a decision procedure $[\mathrm{V}]$. A discrete time Markov chain is a random procedure that undertakes transitions from one state space to another state space. It must hold a "memory lessly", in which the chances of the next state depend on the current state not on the system of events that goes before. This case of the Memory lessness is called Markov property (MP).In real world processes, statistical models MC have many applications. A MC is a stochastic procedure with the MP. The term "MC" refers to the arrangement of arbitrary variables such a procedure exchanges through, with the MP significant sequential dependence only between neighboring periods. It can thus be used for describing systems that follow a chain of linked events, where what happens next depends only on the current state of the system. Different types of MP are selected as "MC" in the previous literature. Generally the stretch is kept for a process with a discrete set of times is called discrete time Markov chain [VI]. MC refers to a continuous deprived of explicit indication. Although the time is taken as a discrete parameter, there is no restriction on the state space in the MC, the period may mention to a method on a random state space [VIII]. Although in MC applications finite or infinite states are considered as discrete state spaces that have more statistical analysis, moreover time catalogue and state space restrictions, a lot of disparities additions and simplifications are founded. In economics, inflation is a continued fluctuation in the general price level of goods and services in an economy with respect to a period of time. Inflation delivers important vision on the national economy and occurs in any economy but with a different rate and strength. Inflation in the price of Food is a highest issue being handled by emerging states a like Pakistan. This indicator use excessive quantity of compression with the economic circumstances of any state [VII]. The movable economic and financial policies of the government of Pakistan have caused in upgrading in numerous macroeconomic factors with Gross Domestic Product (GDP) growth in several years. For example in 1960s, 1980s and the few centuries of the first era of the $21^{\text {st }}$ century, this persisted above 6 percent during 2004-06. Despite this impressive performance of the economy, some worrisome factors have also seemed on this section. The most substantial of these indicators is inflation, which persisted in dual number in $70 \mathrm{~s}$ and also in last numerous years [I].

\section{Methodology}

A growth in import prices was also cause a salient indicator in producing inflation. Correspondingly, some researchers suspected that indirect taxes are the main reasons of inflation. The Wheat Support Price (WSP) recognized as an important element of inflation in Pakistan (Khan and Qasim, 1996). For checking the variability in the inflation rates, the inflation rate data is taken on monthly basis form July-2000 to April-2015 from state bank of Pakistan. Then differentiate inflation rate into 3 states, if rate $<=0$ then it categorize as "Deflation", if $<=1$ then 
it categorize as "Creep", if $>1$ define as "Normal" inflation. A Discrete Time Markov Chain (DTMC) is a sequence of random variables $X_{1}, X_{2}, X_{3}, \ldots X_{n}$, characterized by the Markov property. The Markov property states that the distribution of the forthcoming state $X_{n+1}$ depends only on the current state $X_{\mathrm{n}}$ and doesn't depend on the previous ones $X_{n-1}, X_{n-2}, \ldots, X_{1}$.

$$
P\left(X_{n+1}=x_{n+1} / X_{1}=x_{1}, X_{2}=x_{2}, \ldots, X_{n}=x_{n}\right)=P\left(X_{n+1}=x_{n+1} / X_{n}=x_{n}\right)
$$

The set of possible states $S=\left\{S_{1}, S_{2}, S_{3}, \ldots, S_{n}\right\}$ of $X_{n}$ can be finite or countable and it is named the state space of the chain (Ching.Wet al., 2008).Firstly build a Markov chain model for a pragmatic definite data sequence, adopt the following canonical form representation:

$$
X_{0}=(0,1,0)^{T} X_{1}=(1,0,0)^{T} X_{2}=(0,0,1)^{T} \ldots \quad X_{n}=(0,1,0)^{T}
$$

For $\mathrm{X}_{0}=2, \mathrm{X}_{1}=1, \mathrm{X}_{2}=3 \ldots \mathrm{X}_{\mathrm{n}}=2$.

To estimate the transition probability matrix for the above perceived Markov chain, considered the following simple measures. By totaling the transition frequency from State ito State jin the arrangement, build the transition frequency matrix $\mathrm{N}$ then the transition probability matrix $\mathrm{P}$ for the arrangement as follows:

$$
\left.\begin{array}{rll}
\frac{n_{11}}{n_{1 j}} & \frac{n_{12}}{n_{1 j}} & \frac{n_{13}}{n_{1 j}} \\
P=\frac{n_{21}}{n_{2 j}} & \frac{n_{22}}{n_{2 j}} & \frac{n_{23}}{n_{2 j}} \\
\frac{n_{31}}{n_{3 j}} & \frac{n_{32}}{n_{3 j}} & \frac{n_{33}}{n_{3 j}}
\end{array}\right]
$$

After making the transition matrix check the distribution of the states by using different initial states and check the stability situation after $n$ generation of distribution of the states. In other words to find out the stationary distribution and identifying absorbing and transient states. For statistical analysis simulating a random sequence from an underlying DTMC. Then checks the estimates by using two methods, maximum likelihood, and maximum likelihood with Laplace smoothing.

The maximum likelihood estimator (MLE) of the $p_{i j}$ entry, where the $\mathrm{n}_{\mathrm{ij}}$ element consists in the number sequences $\left(X_{t}=S_{i} ; X t+1=S_{j}\right)$ found in the sample, that is

$$
\hat{p}^{M L E}{ }_{i j}=\frac{n_{i j}}{\sum_{u=1}^{k} n_{i u}}
$$

The Laplace smoothing approach is a variation of the MLE. For tackle the variation in MLE, Laplace smoothing approach will be used. Where the $n_{i j}$ is substituted by $n_{i j}+\propto$. Here $\propto$ an arbitrary positive stabilizing parameter.

$$
\hat{p}_{i j}^{L S}=\frac{n_{i j}+\propto}{\sum_{u=1}^{k}\left(n_{i u}+\propto\right)}
$$

\section{Results}

Table 1: Transition Probability Matrix

\begin{tabular}{|l|l|l|l|}
\hline & Creeping & Trotting & Galloping \\
\hline Creeping & 0.4823529 & 0.3176471 & 0.2000000 \\
\hline Trotting & 0.4137931 & 0.4137931 & 0.1724138 \\
\hline Galloping & 0.5588235 & 0.2352941 & 0.2058824 \\
\hline
\end{tabular}

This matrix show that the probability of inflation rate from creeping to creeping is $48.23 \%, 20 \%$ creeping to galloping and $31.76 \%$ of creeping to trotting. Similarly the probabilities of inflation rate going from galloping to creeping is $55.88 \%, 23.52 \%$ from galloping to trotting, and $20.58 \%$ from galloping to galloping. The probability inflation rate of going from trotting to creeping is $41.37 \%$, from trotting to trotting is $41.37 \%$, and $17.24 \%$ from trotting to galloping inflation.

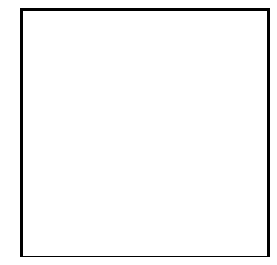

Figure 1: I-graph of Transition Probability Matrix.

For the graphical presentation of transition matrix in Markov chain model igraph is used, now according to the graph we can easily explain the probability of inflation rate moves from one state to another state. Let Creeping $=\mathrm{C}$, Galloping $=\mathrm{G}$, Trotting $=\mathrm{T}$. this graph shows that the occurrence of inflation rate from $\mathrm{C}$ to $\mathrm{C}$ is $48 \%, 20 \%$ creeping to galloping and $32 \%$ creeping to trotting. The probability of $\mathrm{G}$ to $\mathrm{C}$ is $56 \% \mathrm{G}$ to $\mathrm{G}$ is $21 \%$ and $24 \%$ 
chances of inflation rate from $\mathrm{G}$ to $\mathrm{T}$ inflation. Similarly the chances of inflation rate from $\mathrm{T}$ to $\mathrm{C}$ is $41 \%, 17 \%$ from $\mathrm{T}$ to $\mathrm{G}$ and $41 \%$ chance of inflation rate moves from $\mathrm{T}$ to $\mathrm{T}$.

\section{III.I. Equilibrium Distribution of Transition Matrix}

First chose $[1,0,0]$ this initial state in which $1^{\text {st }}$ state of inflation rate is present others are absent then calculate its initial probability vector which is $[0.4823529,0.20000 .3176471]$. This is also called the generation of probability vector which explains that there are $48.23 \%$ probability of creeping state and $20 \%$ probability of galloping and $31.76 \%$ probability of trotting state. The entire probability vectors are generated by this formula.

\section{III.II. Equilibrium Distribution of Transition Matrix}

First chose $[1,0,0]$ this initial state in which $1^{\text {st }}$ state of inflation rate is present others are absent then calculate its initial probability vector which is $X_{0}=[0.4823529,0.20000 .3176471]$. This is also called the generation of probability vector which explains that there are $48.23 \%$ probability of Creeping state and $20 \%$ probability of Galloping and $31.76 \%$ probability of Trotting state. The entire probability vectors are generated by $X_{0} * P^{n}$ this formula.

Table 2: After checking the $\mathrm{N}^{\text {th }}$ generations

\begin{tabular}{|l|l|l|l|}
\hline After generation N & Creeping & Galloping & Trotting \\
\hline 0 & 0.4823529 & 0.2 & 0.3176471 \\
\hline 1 & 0.4758692 & 0.1924138 & 0.331717 \\
\hline 2 & 0.4743244 & 0.191981 & 0.3336945 \\
\hline 3 & 0.4741558 & 0.1919239 & 0.3339203 \\
\hline 4 & 0.4741359 & 0.1919174 & 0.3339467 \\
\hline 5 & 0.4741336 & 0.1919166 & 0.3339498 \\
\hline 6 & 0.4741333 & 0.1919165 & 0.3339501 \\
\hline 7 & 0.4741333 & 0.1919165 & 0.3339502 \\
\hline 8 & 0.4741333 & 0.1919165 & 0.3339502 \\
\hline 9 & 0.4741333 & 0.1919165 & 0.3339502 \\
\hline
\end{tabular}

By using this initial probability vector generates next probability vectors and checks the stability in which generation inflation states probability vectors are stable. After $7^{\text {th }}$ generation the probability vector of inflation states shows the stable chances. We can be see it from the above table the probability vectors of $7^{\text {th }}, 8^{\text {th }}$ and $9^{\text {th }}$ generation are stable at specific probability vector, which explain that there are $47.41 \%$ chance of Creeping, $19.19 \%$ of Galloping and $33.39 \%$ of Trotting state. All the next generations will show the same chances of occurrences of the inflation states, we may generate it into $10^{\text {th }}, 11^{\text {th }}$ times or so on many times.

\section{III.III. Conditional Distributions}

Conditional distribution is used for checking the probability of different states of inflation rate if any one of state is given. In the conditional distribution of inflation states, given that current inflation state is Trotting.

Table 3: Conditional Distributions

\begin{tabular}{|l|l|l|}
\hline Creeping & Galloping & Trotting \\
\hline 0.5588235 & 0.2352941 & 0.2058824 \\
\hline
\end{tabular}

There are $55.88 \%$ chances of Creeping, $23.52 \%$ chances of Galloping and $20.58 \%$ of Galloping when current state is Trotting.

The conditional distribution of inflation states, given that current inflation state is Galloping.

Table 4 : Conditional Distributions

\begin{tabular}{|l|l|l|}
\hline Creeping & Galloping & Trotting \\
\hline 0.4137931 & 0.4137931 & 0.1724138 \\
\hline
\end{tabular}

There are $41.37 \%$ chances of Creeping, 41.37 chances of Galloping and $17.24 \%$ of normal when current state is Trotting.

The conditional distribution of inflation states, given that current inflation state is Creeping.

Table 5: Conditional Distributions

\begin{tabular}{|l|l|l|}
\hline Creeping & Galloping & Trotting \\
\hline 0.4823529 & 0.3176471 & 0.200000 \\
\hline
\end{tabular}

There are $48.23 \%$ chances of Creeping, $31.7 \%$ chances of Galloping and $20 \%$ of Galloping when current state is Trotting.

\section{III.IV. Steady state}

If the Markov chain is a time homogeneous Markov chain, so that the process is described by a single, time independent matrix, $p_{i j}$ then the vector $\pi$ is called a stationary distribution. Note that there is no assumption on the starting distribution; the chain converges to the stationary distribution regardless of where it begins. Such $\pi$ is 
called the equilibrium probability distribution of the Markov chain.

Here the stationary distribution for the states of inflation is

$$
\pi=[0.4741333,0.1919165 .0 .3339502]
$$

This vector explains that there are $47.41 \%$ chance of Creeping, $19.19 \%$ of Galloping and $33.39 \%$ of Trotting state.

IV Maximum Likelihood estimation:

Table 6: MLE Transition Matrix

\begin{tabular}{|l|l|l|l|}
\hline & Creeping & Galloping & Trotting \\
\hline Creeping & 0.4736842 & 0.1842105 & 0.3421053 \\
\hline Galloping & 0.4805195 & 0.2727273 & 0.2467532 \\
\hline Trotting & 0.3111111 & 0.2074074 & 0.4814815 \\
\hline
\end{tabular}

This transition matrix show that the occurrence of inflation rate from Creeping to Creeping is $47.36 \%, 18.42 \%$ Creeping to Galloping and $34.21 \%$ Creeping to Trotting. The probability of Galloping to Creeping is $48.05 \%$, Galloping to Galloping is $27.27 \%$ and $24.67 \%$ chances of inflation rate from Galloping to Trotting inflation rate. Similarly the chances of inflation rate from Trotting to Creeping is $31.11 \%, 20.74 \%$ from Trotting to Galloping and $48.14 \%$ chance of inflation rate moves from Trotting to Trotting inflation rate.

\section{IV.I. Equilibrium Distribution of MLE}

First chose $[1,0,0]$ this initial state in which $1^{\text {st }}$ state of inflation rate is present others are absent then calculate its initial probability vector which is $X_{0}=[0.4736842,0.34210530 .1842105]$. This is also called the generation of probability vector which explains that there are $47.36 \%$ probability of Creeping state and $18.42 \%$ probability of Trotting and $27.41 \%$ probability of Galloping state. The entire probability vectors are generated by $X_{0} * P^{n}$ this formula.

Table7: After checking the $\mathrm{N}^{\text {th }}$ generations

\begin{tabular}{|l|l|l|l|}
\hline After generation N & Creeping & Galloping & Trotting \\
\hline 0 & 0.4736842 & 0.3421053 & 0.1842105 \\
\hline 1 & 0.4193262 & 0.3722218 & 0.208452 \\
\hline 2 & 0.4763518 & 0.1926844 & 0.3309639 \\
\hline 3 & 0.4743968 & 0.1920034 & 0.3335998 \\
\hline 4 & 0.474164 & 0.1919267 & 0.3339093 \\
\hline 5 & 0.4741369 & 0.1919177 & 0.3339454 \\
\hline 6 & 0.4741337 & 0.1919167 & 0.3339496 \\
\hline 7 & 0.4741334 & 0.1919165 & 0.3339501 \\
\hline 8 & 0.4741333 & 0.1919165 & 0.3339502 \\
\hline 9 & 0.4741333 & 0.1919165 & 0.3339502 \\
\hline 10 & 0.4741333 & 0.1919165 & 0.3339502 \\
\hline
\end{tabular}

By using this initial probability vector generates next probability vectors and checks the stability in which generation inflation states probability vectors are stable. After $7^{\text {th }}$ generation the probability vector of inflation states shows the stable chances. We can be see it from the Table-4.12 the probability vectors of $8^{\text {th }}, 9^{\text {th }}$ and $10^{\text {th }}$ generation are stable at specific probability vector, which explain that there are $47.41 \%$ chance of Creeping, $19.19 \%$ of Galloping and $33.39 \%$ of Trotting state. All the next generations will show the same chances of occurrences of the inflation states, we may generate it into $11^{\text {th }}, 12^{\text {th }}$ times or so on many times.

\section{Maximum Likelihood LAPLACE Smoothing :}

Table 8: Transition Matrix of Laplace Smoothing:

\begin{tabular}{|l|l|l|l|}
\hline & Creeping & Galloping & Trotting \\
\hline Creeping & 0.4736565 & 0.1842400 & 0.3421035 \\
\hline Galloping & 0.4804622 & 0.2727509 & 0.2467870 \\
\hline Trotting & 0.3111160 & 0.2074354 & 0.4814486 \\
\hline
\end{tabular}

This transition matrix show that the occurrence of inflation rate from Creeping to Creeping is $47.36 \%, 18.42 \%$ Creeping to Galloping and $34.21 \%$ Creeping to Trotting. The probability of Galloping to Creeping is $48.04 \%$, Galloping to Galloping is $27.27 \%$ and $24.67 \%$ chances of inflation rate from Galloping to Trotting inflation. Similarly the chances of inflation rate from Trotting to Creeping is $31.11 \%, 20.74 \%$ from Trotting to Galloping and $48.14 \%$ chance of inflation rate moves from Trotting to Trotting.

\section{V.I. Equilibrium Distribution of Laplace Smoothing}

First chose $[1,0,0]$ this initial state in which $1^{\text {st }}$ state of inflation rate is present others are absent then calculate its 
initial probability vector which is $X_{0}=\left[\begin{array}{lll}0.4736565 & 0.3421035 & 0.18424\end{array}\right]$. This is also called the generation of probability vector which explains that there are $18.42 \%$ probability of Trotting state and $47.36 \%$ probability of Creeping and $34.21 \%$ probability of Galloping state. The entire probability vectors are generated by $X_{0} * P^{n}$ this formula.

Table 9: After checking the $\mathrm{N}^{\text {th }}$ generations

\begin{tabular}{|l|l|l|l|}
\hline After generation N & Creeping & Galloping & Trotting \\
\hline 0 & 0.4736565 & 0.3421035 & 0.18424 \\
\hline 1 & 0.4193047 & 0.3722128 & 0.2084825 \\
\hline 2 & 0.4145757 & 0.3740977 & 0.2113266 \\
\hline 3 & 0.4142887 & 0.3740893 & 0.2116221 \\
\hline 4 & 0.4142921 & 0.37406 & 0.211648 \\
\hline 5 & 0.4142971 & 0.3740534 & 0.2116496 \\
\hline 6 & 0.4142981 & 0.3740523 & 0.2116496 \\
\hline 7 & 0.4142983 & 0.3740522 & 0.2116496 \\
\hline 8 & 0.4142984 & 0.3740522 & 0.2116496 \\
\hline 9 & 0.4142984 & 0.3740522 & 0.2116496 \\
\hline 10 & 0.4142984 & 0.3740522 & 0.2116496 \\
\hline
\end{tabular}

By using this initial probability vector generates next probability vectors and checks the stability in which generation inflation states probability vectors are stable. After $7^{\text {th }}$ generation the probability vector of inflation states shows the stable chances. We can be see it from the above table the probability vectors of $8^{\text {th }}, 9^{\text {th }}$ and $10^{\text {th }}$ generation are stable at specific probability vector, which explain that there are $21.16 \%$ chance of Trotting, $41.42 \%$ of Creeping and $37.40 \%$ of Galloping state. All the next generations will show the same chances of occurrences of the inflation states, we may generate it into $11^{\text {th }}, 12^{\text {th }}$ times or so on many time.

\section{Comparison:}

Table 10: Comparison between MLE \& Laplace Smoothing

\begin{tabular}{|l|l|l|}
\hline Inflation States & MLE & Laplace Smoothing \\
\hline Trotting & $33.39 \%$ & $21.16 \%$ \\
\hline Creeping & $47.41 \%$ & $41.42 \%$ \\
\hline Galloping & $19.19 \%$ & $37.40 \%$ \\
\hline
\end{tabular}

It is illustrated that Laplace smoothing estimates are used for those counts that are unseen in the counting. We can see from Table-10 that in Trotting state MLE estimate is 33.39\% but Laplace Smoothing is $21.16 \%$. In MLE estimates $47.41 \%$ chances of Creeping and 19.19\% chance of Galloping inflation rate but when we checks Laplace Smoothing estimates that are shown in the table-10 that the probability of Creeping and Galloping inflation rates are $41.42 \%$ and $37.40 \%$. In Laplace Smoothing, Trotting and Creeping inflation rates decreases as compare to MLE estimates and probability of Galloping inflation rate is increased as compare to MLE, Because a little portion of the counts move to Trotting state in Laplace Smoothing that why the chances of Galloping state is more then as compare to MLE estimates.

\section{VII: Conclusion}

The current study based on Markov Chain Model, core purpose of present study was according to Markov Chain Model related to the economics features. For this study Macroeconomic indicator inflation rate is used and found that it is changing since several years. It has a foremost outcome on the progress and economic development of any country. Study data was form July 2000 to April 2015. we categorized our data into three different states that are "Trotting", "Creeping" and "Galloping" inflation rate as per as the key features of Markov Chain Model. First of all Checked the Transition count and Transition Matrix where it is easily to checked the progress chances of one state in comparison to another state. Equilibrium distribution is very important for the Markovian Transition Matrix its clarifies the stability point for the model whether it is checked on different initial vectors. Conditional distribution explained about the chances of other states if anyone is fixed that we know about it already then check the chances of there. The "markov chain" I graph R software package that helped for the Graphical presentation of the model. Transition Matrix are Smoothing Techniques that provided by using Maximum Likelihood and Maximum Likelihood with Laplace. Equilibrium distribution is obtain of the model separately by mutually techniques and checks the stability point of these distributions by taking different initial vectors. At the last the comparison is take place in stability points between two techniques. Estimates obtain on the fact and figures of Laplace Smoothing technique are reliable because it control the variation on the Maximum Likelihood estimates. It takes all the uncounts that are not counted in using Maximum Likelihood estimation. 


\section{REFERENCES}

I. $\quad$ Ahmed Q.M., Muhammad S.D., Noman M., Lakhan G.R. (2014).Determinants of recent inflation in Pakistan: Revisit. Pakistan Journal of Commerce and Social Sciences 8:170-184.

II. $\quad$ Ching W.(2001).Iterative Methods for Queuing and Manufacturing Systems. Springer-Verlag London Ltd., London.

III. Ching W., Fung E., Ng M. (2003).A higher-order Markov model for the Newsboy's problem. Journal of the Operational Research Society 54:291-298.

IV. $\quad$ Ching W.K., Fung E.S., Ng M.K. (2002).A multivariate Markov chain model for categorical data sequences and its applications in demand predictions. IMA Journal of Management Mathematics 13:187199.

V. Ching, W., Ng, M. \& Fung, E. (2008). Higher-order multivariate Markov chains and their applications. Linear Algebra and its Applications428, pp.492-507.

VI. Everitt,B.S. (2002). The Cambridge Dictionary of Statistics. CUP. ISBN 0-521-81099-X.

VII. Joiya S.A., Shahzad A. (2013).Determinants of High Food Prices. Pakistan Economic and Social. Review 51:93-107.

VIII. Meyn, S. Sean P., and Richard L. Tweedie. (2009). Markov chains and stochastic stability. Cambridge University Press. (Preface, p. iii). 